\title{
Heart rate turbulence as risk-predictor after myocardial infarction
}

\author{
Christine S. Zuern ${ }^{1}$, Petra Barthel ${ }^{2}$ and Axel Bauer ${ }^{1}$ * \\ 1 Innere Medizin III (Kardiologie), Eberhard-Karls-Universität Tübingen, Tübingen, Germany \\ ${ }^{2}$ Medizinische Klinik, Technische Universität München University, München, Germany
}

\section{Edited by:}

Heikki Veli Huikuri, University of Oulu,

Finland

Reviewed by:

Juha Koskenvuo, University of Turku, Finland

Phyllis Kravet Stein, Washington

University School of Medicine, USA

${ }^{*}$ Correspondence:

Axel Bauer, Innere Medizin III,

Eberhard-Karls-Universität Tübingen,

Otfried-Müller-Str. 10, 72076

Tübingen, Germany.

e-mail:axel.bauer@med.

uni-tuebingen.de
Heart rate turbulence (HRT) is the baroreflex-mediated short-term oscillation of cardiac cycle lengths after spontaneous ventricular premature complexes. HRT is composed of a brief heart rate acceleration followed by a gradual heart rate deceleration. In high risk patients after myocardial infarction (MI) HRT is blunted or diminished. Since its first description in 1999 HRT emerged as one of the most potent risk factors after MI. Predictive power of HRT has been studied in more than 10,000 post-infarction patients. This review is intended to provide an overview of HRT as risk-predictor after MI.

Keywords: autonomic function, heart rate turbulence, myocardial infarction, risk stratification, sudden death

\section{INTRODUCTION}

Despite significant advances in interventional and medical therapy late mortality after myocardial infarction (MI) is still high. A substantial number of these late deaths occur suddenly, potentially preventable by an implantable cardioverter-defibrillator (ICD). Randomized multicenter trials have shown that mortality can be reduced in post-infarction patients at high risk for death by 20 54\% (Moss et al., 1996, 2002; Buxton et al., 1999; Bristow et al., 2004; Bardy et al., 2005). Current guidelines recommend ICD implantation in patients characterized by a compromised left ventricular ejection fraction (LVEF 30-35\%) which is considered to be the gold standard in risk prediction (Gregoratos et al., 2002; Zipes et al., 2006).

However, clinical studies have consistently shown that the criterion of a reduced LVEF is neither sensitive nor specific. It lacks of sensitivity as approximately $2 / 3$ of deaths in post-infarction patients occur in patients with LVEF $>35 \%$ who are not covered by the criterion of reduced LVEF (Myerburg et al., 1997, 1998; Huikuri et al., 2001; Buxton, 2003). It also lacks of specificity as 11 ICDs have to be implanted to save one life (Camm et al., 2007). The majority of ICD recipients will never receive an adequate shock. Therefore, development of additional risk stratification strategies is urgently needed.

Twelve years ago, an electrocardiographic phenomenon later on termed "heart rate turbulence (HRT)" has been described (Schmidt et al., 1999). At that time, it has been firstly recognized that in healthy persons spontaneous ventricular premature complexes (VPC) are followed by a characteristic short-term oscillation of heart rate. The oscillation is composed of a brief heart rate acceleration followed by a gradual heart rate deceleration before returning to baseline. As post-ectopic changes of cycle lengths are in the range of milliseconds and masked by heart rate variability of other origin it can only be visualized by signal averaging.
Very early it became clear that post-infarction patients at increased risk for adverse events showed different postextrasystolic patterns of heart rate. In high risk patients the typical HRT response is blunted or entirely missing. Within the last decade, HRT emerged as one of the most potent ECG based risk predictors. Several large-scale studies have demonstrated its strong prognostic power in post-infarction patients. This review is intended to provide a review of HRT as risk-predictor in post-infarction patients.

\section{MEASUREMENT OF HEART RATE TURBULENCE}

Heart rate turbulence is obtained from standard 24-h Holter recordings with a minimum temporal resolution of $128 \mathrm{~Hz}$ which allow for accurate determinations of RR intervals and beat classifications. In contrast to other techniques such as T-wave alternans no specific electrodes or other equipments are needed. The technical assessment of HRT has been described in details elsewhere (Bauer et al., 2008). Briefly, the RR intervals surrounding spontaneous VPCs are averaged in order to obtain a so-called local tachogram demasking the average pattern of sinus RR intervals surrounding VPCs (Figure 1). VPCs used for HRT computation need to fulfill certain criteria with respect to prematurity and compensatory pause. Details regarding these filter criteria have been described elsewhere (Bauer et al., 2008).

The two phases of HRT are quantified by the two parameters turbulence onset (TO) and turbulence slope (TS).

Turbulence onset is calculated as follows:

$\mathrm{TO}=\frac{\left(\mathrm{RR}_{1}+\mathrm{RR}_{2}\right)-\left(\mathrm{RR}_{-2}+\mathrm{RR}_{-1}\right)}{\left(\mathrm{RR}_{-2}+\mathrm{RR}_{-1}\right)} \times 100[\%]$

whereas $R_{-2}$ and $R_{-1}$ are the two $R R$ intervals immediately before the VPC coupling interval. $R_{1}$ and $R_{2}$ are the 


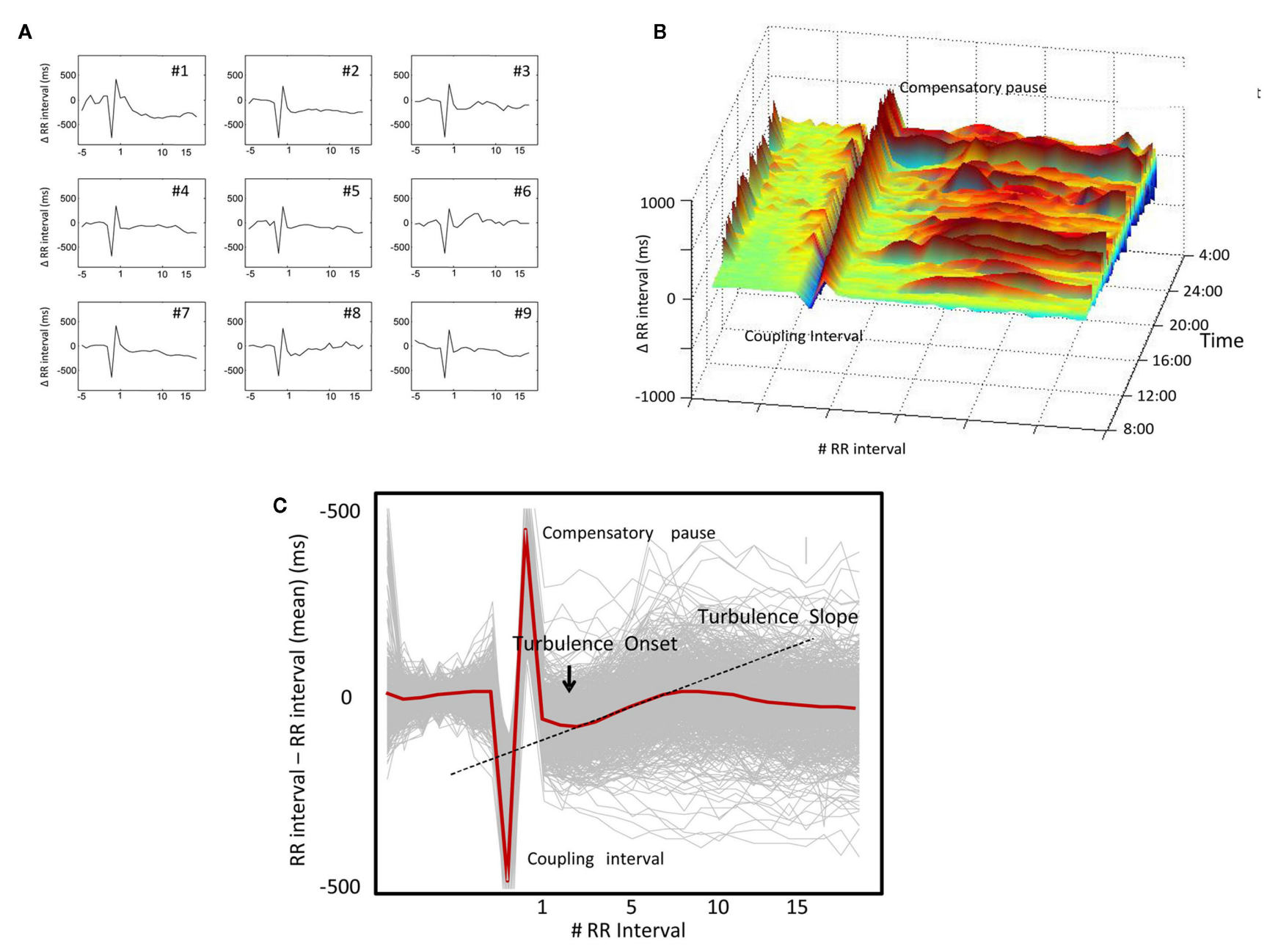

FIGURE 1 | Assessment of heart rate turbulence (HRT) from 24-h Holter recordings: $(A)$ high variability in single "local tachograms" surrounding randomly selected ventricular premature complexes (VPCs); (B) high circadian variability in single local tachograms observed over $24 \mathrm{~h}$; although typical HRT response can be suggested from this graph; (C) smooth HRT pattern (red curve) after signal averaging of single tachograms (gray curves). Quantification of HRT by turbulence onset and turbulence slope (see text for explanation). two RR intervals which immediately follow the compensatory pause. TS is defined as the maximum positive regression slope assessed over any five consecutive sinus RR intervals within the first $15 \mathrm{RR}$ intervals following the VPC. Hence, in normal subjects, the initial acceleration of sinus rate after the VPC is characterized by negative TO and the following heart rate deceleration is characterized by positive TS. TO $<0 \%$ and TS $>2.5 \mathrm{~ms} / \mathrm{RR}$ interval are considered normal (Bauer et al., 2008).

For use of risk stratification in different patient populations, HRT is usually divided into three categories: (1) HRT category 0 is defined as normal TO and normal TS, (2) HRT category 1 means that either TO or TS are abnormal, and (3) HRT category 2 is characterized by both abnormal TO and TS. If no sufficient VPC tachograms are recorded and patients are otherwise in sinus rhythm, HRT is classified as category 0 since those patients were shown to have equally good prognosis as patients with normal HRT (Barthel et al., 2003). As this was only shown for post-infarction patients, this approach might not be valid if other pathologies (e.g., heart failure) are considered.

The HRT software is commercially available on GE and Getemed Holter systems. However, as the algorithms have been published in detail (www.h-r-t.com) HRT can also be obtained from the series of RR intervals by a custom-made software.

\section{PHYSIOLOGY OF HEART RATE TURBULENCE}

When the first clinical studies of HRT in risk prediction have been published the exact physiological mechanisms behind HRT were largely unknown (Schmidt et al., 1999; Bauer and Schmidt, 2007). The (patho)physiological mechanisms behind HRT are complex and involve both branches of the autonomic nervous system. In their work, Wichterle et al. (2006) provide an excellent review of HRT physiology. The VPC induces a transient drop of arterial blood pressure which leads to an activation of the baroreceptors. Vagal activity is abruptly withdrawn resulting in an almost 
immediate shortening of RR interval cycle lengths (as measured by TO). However, also the sympathetic system reacts (Segerson et al., 2007). Increased sympathetic activity results in a gradual increase of vascular resistance and systolic arterial blood pressure. As consequence, vagal activity reestablishes and cycle lengths prolong (as measured by TS). Importantly, HRT requires an intact interplay of both, vagal and sympathetic systems. Absence of normal HRT can be caused by an alteration in one of the systems (Wichterle et al., 2006).

\section{HRT STUDY POPULATIONS}

Evidence of HRT as risk-predictor in post-infarction patients is based on five retrospective and five prospective studies including a total of more than 10,000 patients. Study characteristics are summarized in Tables 1 and 2.

Heart rate turbulence was originally developed in a small dataset comprising of 100 patients suffering from coronary artery disease and subsequently validated in a blinded fashion in the cohorts of the MPIP study $(n=577)$ and the placebo arm of the EMIAT study ( $n=614$; Schmidt et al., 1999). Two years later, Ghuran et al. (2002) tested the predictive power of HRT in the dataset of the ATRAMI study $(n=1,212)$ which was originally designed to assess the prognostic power of baroreflex sensitivity. Another 3 years later, predictive power of HRT was also tested in the dataset of the CAST study ( $n=744$; Hallstrom et al., 2005). The FINGER study combined a Finish and German post-infarction population (Barthel et al., 2003; Huikuri et al., 2003) to specifically address the question whether HRT predicts sudden death (Makikallio et al., 2005).

In 2003, the results of the first prospective study ISAR-HRT $(n=1,455)$ were published which was designed to validate the prognostic value of HRT in a large cohort of post-infarction patients receiving contemporary treatment (Barthel et al., 2003). The REFINE study $(n=322)$ published 2007 was designed to assess the predictive value of a combination of several risk predictors including HRT as well as the time of their assessment after acute MI (Exner et al., 2007). In 2009, the results of the largest prospective HRT study were published. ISARRISK tested the prognostic value of a combination of HRT and deceleration capacity (Bauer et al., 2006a) in post-infarction patients with preserved LVEF (Bauer et al., 2009a). Deceleration capacity is an integral measure of all deceleration related modulations of heart rate observed over $24 \mathrm{~h}$ and thus, most presumably, a measure of tonic vagal activity. The CARISMA study $(n=312)$ deserves special attention as loop recorders have been implanted in all patients to specifically address the endpoint of severe arrhythmic events (Huikuri et al., 2009). Very recently, the results of ISAR-SWEET have been published

Table 1 | Retrospective studies (or sub-studies) investigating heart rate turbulence as a post-infarction risk-predictor.

\begin{tabular}{|c|c|c|c|c|c|}
\hline & $\begin{array}{l}\text { MPIP } \\
\text { (Schmidt } \\
\text { et al., 1999) }\end{array}$ & $\begin{array}{l}\text { EMIAT } \\
\text { (Schmidt } \\
\text { et al., 1999) }\end{array}$ & $\begin{array}{l}\text { ATRAMI } \\
\text { (Ghuran et al., } \\
\text { 2002) }\end{array}$ & $\begin{array}{l}\text { CAST } \\
\text { (Hallstrom } \\
\text { et al., 2005) }\end{array}$ & $\begin{array}{l}\text { FINGER } \\
\text { (Makikallio } \\
\text { et al., 2005) }\end{array}$ \\
\hline Number of patients & 577 & 614 & 1,212 & 744 & 2,130 \\
\hline $\begin{array}{l}\text { Year of publication of } \\
\text { original study }\end{array}$ & 1983 & 1997 & 1998 & 1991 & 2003 \\
\hline Inclusion criteria* & $\begin{array}{l}\mathrm{MI} \leq 4 \text { weeks, } \\
\text { age } \leq 70 \text { years }\end{array}$ & $\begin{array}{l}\mathrm{MI} \leq 4 \text { weeks, age } \\
\leq 75 \text { years, LVEF } \leq 40 \%\end{array}$ & $\begin{array}{l}\mathrm{MI} \leq 4 \text { weeks, } \\
\text { age } \leq 80 \text { years }\end{array}$ & $\mathrm{Ml} \geq 6 \mathrm{VPC} / \mathrm{h}$ & $\begin{array}{l}\mathrm{MI} \leq 4 \text { weeks, age } \\
\leq 75 \text { years }\end{array}$ \\
\hline Follow-up (months) & 22 & 21 & 20 & 55 & 33 \\
\hline Endpoint & Mortality & Mortality & Cardiac mortality ${ }^{\dagger}$ & Mortality & Sudden death \\
\hline Endpoints reached (\%) & 13 & 14 & 4 & $29^{\ddagger}$ & 2 \\
\hline $\begin{array}{l}\text { Time of HRT } \\
\text { assessment after MI }\end{array}$ & 2nd week & 2nd to 3rd week & 2nd to 4 th week & 10 weeks & 2nd week \\
\hline Treatment of acute MI & None & $60 \%$ Lysis & $63 \%$ Lysis & $28 \%$ Lysis & $70 \% \mathrm{PCl}, 14 \%$ lysis \\
\hline Mean LVEF (\%) & 45 & 30 & 49 & 37 & Not specified \\
\hline Betablockers (\%) & 55 & 32 & 20 & 30 & 94 \\
\hline \multicolumn{6}{|c|}{ UNIVARIATE ANALYSIS } \\
\hline HRT category 2 & $5.0(2.8-8.8)$ & $4.4(2.6-7.5)$ & $6.9(3.1-15.5)$ & Not specified & $4.6(2.6-8.1)^{\|}$ \\
\hline LVEF $\leq 30 \%$ & $4.0(2.5-6.4)$ & $2.2(1.4-3.5)$ & $4.7(2.6-8.3)$ & Not specified & $4.5(2.5-8.0)^{\#}$ \\
\hline \multicolumn{6}{|c|}{ MULTIVARIATE ANALYSIS } \\
\hline HRT category 2 & $3.2(1.7-6.0)$ & $3.2(1.8-5.6)$ & $4.1(1.7-9.8)$ & $20.4(10.2-30.6)^{* *}$ & $2.9(1.6-5.5)$ \\
\hline LVEF $\leq 30 \%$ & $2.9(1.8-4.9)$ & $1.7(1.1-2.7)$ & $3.5(1.8-7.1)$ & Not specified & Not specified \\
\hline
\end{tabular}

* Sinus rhythm was inclusion criterion in all studies.

${ }^{+}$Cardiac Mortality included fatal and non-fatal cardiac arrest.

${ }^{*}$ Cumulative mortality rate only presented for total study population of CAST after 5 years.

"Relative risks presented for turbulence slope $\leq 2.5 \mathrm{~ms} / \mathrm{RR}$ interval.

${ }^{*}$ LVEF was dichotomized at $35 \%$.

** Log of Turbulence Slope corrected for heart rate and VPC count (optimized in CAST data). 
Table 2 | Prospective studies (or sub-studies) investigating heart rate turbulence as a post-infarction risk-predictor.

\begin{tabular}{|c|c|c|c|c|c|}
\hline & $\begin{array}{l}\text { ISAR-HRT } \\
\text { (Barthel et al., } \\
\text { 2003) }\end{array}$ & $\begin{array}{l}\text { REFINE } \\
\text { (Exner et al., } \\
\text { 2007) }\end{array}$ & $\begin{array}{l}\text { ISAR-RISK }{ }^{\ddagger \ddagger} \\
\text { (Bauer et al., } \\
\text { 2009a) }\end{array}$ & $\begin{array}{l}\text { CARISMA } \\
\text { (Huikuri et al., } \\
\text { 2009) }\end{array}$ & $\begin{array}{l}\text { ISAR-Sweet }^{\ddagger \ddagger} \\
\text { (Barthel et al., } \\
\text { 2011) }\end{array}$ \\
\hline Number of patients & 1,455 & 322 & 2,343 & 312 & 481 \\
\hline Inclusion criteria* & $\begin{array}{l}\mathrm{MI} \leq 4 \text { weeks, age } \\
\leq 75 \text { years }\end{array}$ & MI, LVEF <50\% & $\begin{array}{l}\mathrm{Ml} \leq 4 \text { weeks, age } \\
\leq 75 \text { years }\end{array}$ & $\mathrm{MI}<21$ days, LVEF $\leq 40 \%$ & $\begin{array}{l}\mathrm{MI} \leq 4 \text { weeks, age } \\
\leq 80 \text { years, diabetes }\end{array}$ \\
\hline Follow-up (months) & 22 & 47 & 60 & 24 & 60 \\
\hline Endpoint & Mortality & Cardiac death $^{\dagger}$ & Mortality & VF/sustained VT on loop recorder & Mortality \\
\hline Endpoints reached (\%) & 5 & 9 & 8 & 8 & 17 \\
\hline $\begin{array}{l}\text { Time of HRT } \\
\text { assessment after MI }\end{array}$ & 2nd week & $\begin{array}{l}2 \text { nd to } 4 \text { th and } 10 \text { th } \\
\text { to } 14 \text { th week }\end{array}$ & 2nd week & 1 st and 6th week & 2nd week \\
\hline Treatment of acute MI & $90 \% \mathrm{PCl}, 6 \%$ lysis & $45 \% \mathrm{PCl}, 21 \%$ lysis & $92 \% \mathrm{PCl}, 3 \%$ lysis & $14 \% \mathrm{PCl}, 34$ lysis & $89 \% \mathrm{PCl}$ \\
\hline Mean LVEF (\%) & 56 & 47 & 55 & 31 & 51 \\
\hline Betablockers (\%) & 93 & 92 & 94 & 96 & 94 \\
\hline \multicolumn{6}{|c|}{ UNIVARIATE ANALYSIS } \\
\hline HRT category 2 & $11.4(5.7-22.8)$ & $2.9(1.1-7.5)^{\dagger \dagger}$ & $7.5(5.3-10.7)$ & $2.8(1.1-7.2)^{\|}$ & $6.6(3.9-11.0)$ \\
\hline LVEF $\leq 30 \%$ & $7.1(4.2-12.1)$ & $3.3(1.4-7.6)$ & $6.1(4.2-8.7)$ & $1.3(0.5-3.0)^{\#}$ & $4.7(2.8-7.8)$ \\
\hline \multicolumn{6}{|c|}{ MULTIVARIATE ANALYSIS } \\
\hline HRT category 2 & $5.9(2.9-12.2)$ & Not specified & $3.1(2.1-4.6)$ & Not specified & $4.1(2.3-7.2)$ \\
\hline LVEF $\leq 30 \%$ & $4.5(2.6-7.8)$ & Not specified & $3.0(2.0-4.4)$ & Not specified & $2.4(1.4-4.1)$ \\
\hline
\end{tabular}

* Sinus rhythm was inclusion criterion in all studies.

${ }^{+}$Cardiac mortality included fatal and non-fatal cardiac arrest.

"Relative risks presented for turbulence slope $\leq 2.5 \mathrm{~ms} / \mathrm{RR}$ interval.

\#LVEF was dichotomized at $35 \%$.

${ }^{+t}$ HRT category $\geq 1$ vs. 0 tested; HRT was assessed 10-14 weeks after MI.

${ }^{\ddagger}$ ISAR-RISK primarily tested the combination HRT category 2 and abnormal deceleration capacity (Bauer et al., 2006a).

which tested the combination of abnormal HRT and deceleration capacity in diabetic post-infarction patients (Barthel et al., 2011).

\section{RISK PREDICTIVE POWER OF HRT IN POST-INFARCTION PATIENTS}

In all populations, abnormal HRT was a significant and independent predictor of adverse events yielding a relative risk of 2.8-11.4 on univariate and 3.1-5.9 on multivariate analysis.

The single HRT studies substantially differ with respect to study design (retrospective vs. prospective), the primary endpoint investigated (total mortality, cardiac mortality, and sudden death), time of follow-up, time after MI when Holter recordings have been performed, mean LVEF, and treatment of MI.

Heart rate turbulence is generally a very strong predictor in all studies which used total mortality as primary endpoint (which most of the studies did: MPIP, EMIAT, CAST, ISAR-HRT, ISARRISK, ISAR-SWEET). HRT was also a very strong predictor in the ATRAMI study which used a combined endpoint of cardiac mortality and fatal and non-fatal cardiac arrest. Two studies investigated sudden death as primary endpoint, namely the FINGER study and the CARISMA study. While in the FINGER study mode of death was determined anamnestically or with the use of medical recordings, CARISMA had a unique study design: all patients of the CARISMA study underwent implantation of a loop recorder which allowed for a definite assessment of the rhythm at time of death. While HRT was a strong predictor of sudden death in the FINGER study, predictive power of HRT in the CARISMA study was somewhat lower although still significant [relative risk of abnormal TS 2.8 (95\% CI 1.1-7.2), $p=0.038$ ]. It should be noted, however, that in CARISMA only 25 endpoints occurred during a follow-up of 2 years and that only patients with reduced LVEF $(\leq 40 \%)$ have been included. Therefore, conclusions drawn from the CARISMA should not be directly extrapolated to unselected post-infarction populations with no restriction of LVEF.

Time of HRT assessment after acute MI is an important issue. In most HRT studies Holter recordings have been performed early after MI (usually within the first 4 weeks; MPIP, EMIAT, ATRAMI, FINGER, ISAR-HRT, ISAR-RISK, and ISARSWEET). In all of these studies, HRT was a strong and significant predictor of the primary endpoint. However, also in CAST, where risk assessment was performed later after MI, HRT was a strong predictor of death (Hallstrom et al., 2005). In two studies, namely the REFINE and the CARISMA study, risk assessment has been performed at two different time points. In REFINE risk assessment has been performed between the 2nd and the 4 th week as well as between the 10th and the 14th week after MI. In CARISMA, risk assessment has been performed during the first week as well as during the sixth week after MI. In both studies, risk assessment later after MI was superior to risk assessment earlier after MI. It might therefore be concluded, that HRT assessed later after MI might be a better predictor than HRT assessed early after MI. These findings are in 
agreement with the observation that autonomic dysfunction early after MI might recover, and that patients with sustained blunted autonomic function have the worst prognosis (Huikuri et al., 2010).

The single HRT studies cover the whole spectrum of MI treatment eras. In summary, neither acute treatment of MI (conservative, lysis, PCI) nor concomitant medical treatment including betablockers, ACE-inhibitors, or statins seem to affect risk predictive power of HRT. However, it should be noted that effective reperfusion strategies for acute MI which lead to increased myocardial salvage translate into improved autonomic function and better HRT (Bauer et al., 2009b).

Positive predictive accuracies and sensitivities of abnormal HRT for prediction of adverse events strongly depend on the populations and endpoints investigated. Generally, both positive predictive accuracy and sensitivity is in the range of that yielded by LVEF $\leq 30 \%$. Figure 2 exemplarily shows risk stratification by HRT in the largest post-infarction study, the ISARRISK study. Of the 2,343 patients studied, HRT category 2 identified a high risk group of 193 patients (8\%) out of whom 56 patients died. 2,150 patients $(92 \%)$ had normal (HRT category 0 and 1) out of whom 125 patients died. Probability of death within 5 years of follow-up of patients with abnormal HRT (HRT category 2) was $34 \%$. In contrast, the 1,652 patients $(71 \%)$ with completely normal HRT (HRT category 0 ) had a 5 -year mortality of $6 \%$. These figures translate into a positive predictive accuracy of $34 \%$ at a sensitivity level of $31 \%$.

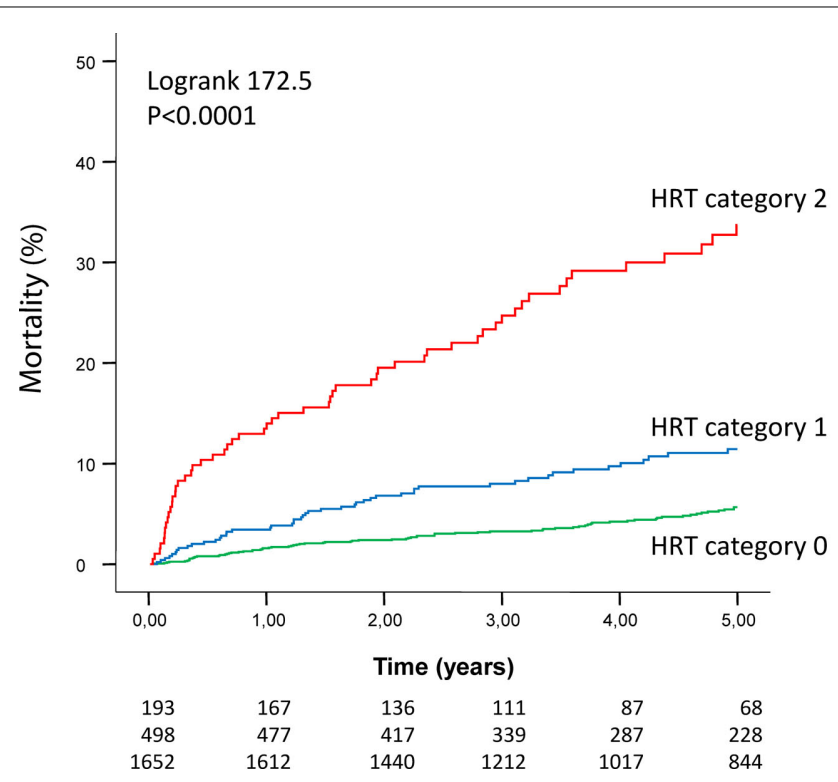

FIGURE 2 | Risk stratification by heart rate turbulence in the ISAR-RISK study (Bauer et al., 2009a). The number of patients of the individual groups involved in the analyses at 0, 1, 2, 3, 4, and 5 years are shown under the graphs. The top and the bottom row corresponds to the upper and bottom Kaplan-Meier curve respectively. For all three comparisons (HRT category 0 vs. HRT category 1, HRT category 0 vs. HRT category 2 , and HRT category 1 vs. HRT category 2 ) the $p$-value was $<0.0001$. (Adapted by permission from Bauer et al., 2009a).

\section{INDEPENDENCY FROM AND COMBINATION WITH OTHER RISK PREDICTORS}

In all studies, predictive value of HRT was independent from that of other risk predictors tested. These included demographic factors and comorbidities (age, gender, presence of diabetes mellitus, and renal insufficiency; Barthel et al., 2011), markers of electrical instability [arrhythmias, T-wave alternans (Exner et al., 2007), late potentials (Bauer et al., 2005), QRS duration (Bauer et al., 2006b)], markers of structural damage (e.g., LVEF), and other markers of autonomic dysfunction (heart rate, heart rate variability, and deceleration capacity; Bauer et al., 2006a, 2009a).

In order to enhance risk predictive power, HRT should be combined with other risk predictors. The ISAR-RISK and the ISAR-SWEET studies investigated the combination of abnormal HRT (HRT category 2 ) with mildly abnormal deceleration capacity $(\leq 4.5 \mathrm{~ms})$ which is a measure of tonic autonomic activity and bases on the processing of RR interval time series by a new mathematical algorithm (Bauer et al., 2006c). For this combination, the term "severe autonomic failure (SAF)" has been introduced. In both, ISAR-RISK and ISAR-SWEET which included 2,343 and 481 patients respectively, SAF proved to be the strongest predictor of death. These findings were confirmed by the results of a recent metaanalysis which analyzed the combined populations of the MPIP, EMIAT, and MRFAT studies ( $n=2,594$; Bauer et al., 2009c). Also the combination of HRT with T-wave alternans is promising as both were independently associated with the primary endpoint in the REFINE study. However, available data do not allow for final conclusions.

Risk stratification by HRT is complementary to risk stratification by LVEF. Only a small proportion of patients with abnormal HRT (category 2) also have LVEF $\leq 30 \%$. Therefore, the strength of HRT lies in the identification of high risk patients in the large group of patients with preserved LVEF ( $>30 \%$ ). Figure 3 shows complimentary risk stratification by HRT and LVEF in the ISARRISK study (Bauer et al., 2009a). The small proportion of patients with both, abnormal HRT and impaired LVEF $(n=40 ; 1.7 \%$ of the study population) had the worst prognosis. Patients with either abnormal HRT ( $n=153 ; 6.5 \%$ of the study population) or impaired LVEF ( $n=80 ; 3.4 \%$ of the study population) had equally poor prognosis. In contrast, patients with normal HRT (category 0 or 1$)$ and LVEF $>30 \%(n=2,070 ; 88.3 \%$ of the study population) had an excellent prognosis. As mentioned above risk stratification by HRT can be further improved by combination with deceleration capacity (Bauer et al., 2009a).

\section{LIMITATIONS OF HEART RATE TURBULENCE}

Several limitations need to be recognized when using HRT as riskpredictor. Firstly, assessment of HRT requires the presence of sinus rhythm. Patients with other rhythms than sinus rhythm such as atrial fibrillation have been excluded in all HRT studies but might be at increased risk. In the Global Utilization of Streptokinase and Tissue plasminogen activator for Occluded coronary arteries (GUSTO-I) trial, $10.5 \%$ of the 41,021 patients had atrial fibrillation during hospitalization (Crenshaw et al., 1997). Further, most HRT studies also excluded elderly patients (age $>75$ years or age $>80$ years). It is well known from the ATRAMI study that autonomic function loses some of its predictive value with increasing 
A

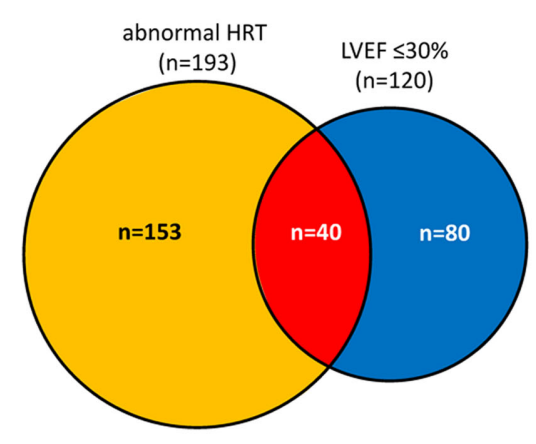

\section{B}

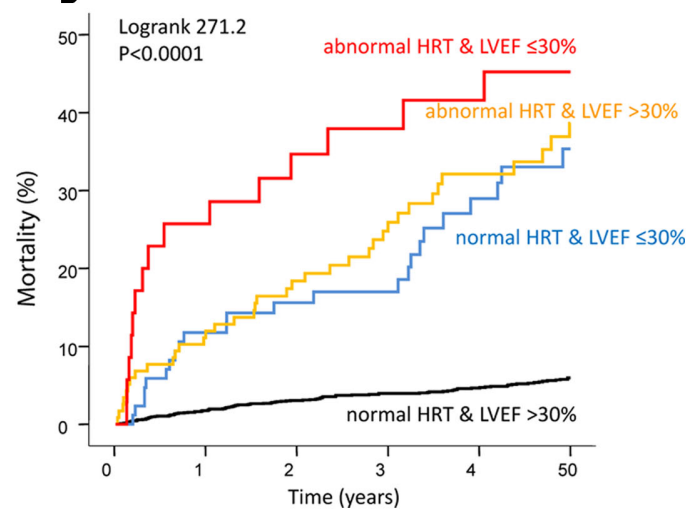

FIGURE 3 | Complimentary risk stratification by HRT and LVEF in the ISAR-RISK study (Bauer et al., 2009a). (A) Distribution of patients with abnormal HRT but LVEF $>30 \%$ ( $n=153$, orange area), abnormal HRT and LVEF $\leq 30 \%(n=40$, red area), and normal HRT but LVEF $\leq 30 \%$ ( $n=80$, blue area). (B) Corresponding 5-year mortality rates of the three groups. Abnormal HRT defined as HRT category 2 (adapted by permission from Bauer et al., 2009 a).

age (La Rovere et al., 1998). Similar observations have been made for HRT in the ISAR-HRT study (Barthel et al., 2005). In contrast, HRT was a significant predictor for sudden death in the population based Cardiovascular Health Study which included adults $\geq 65$ years (average 73 years; Stein et al., 2010). Assessment of HRT also requires presence of VPCs. In most studies, patients without VPCs have therefore been excluded from the analysis (e.g., MPIP, EMIAT, ATRAMI). As shown in the ISAR-HRT study postinfarction patients without VPCs have equally good prognosis as patients with normal HRT (Barthel et al., 2003). Therefore, these patients might be treated as having normal HRT (HRT category 0 ). HRT is usually assessed from full 24-h Holter recordings. Whether HRT derived from shorter recordings provides similar predictive value needs further investigations. A retrospective analysis of HRT in the Multicenter Automatic Defibrillator Implantation Trial 2 that used only 10-min recordings showed the inappropriateness of very short recordings (Berkowitsch et al., 2004). All post-infarction HRT studies included patients early after MI. A large-scale study which investigates the prognostic value of HRT in patients with remote infarction is still missing. This might be of substantial importance as prophylactic ICD implantation in the acute phase of infarction has been generally questioned by the negative results of two randomized ICD trials (Hohnloser et al., 2004; Steinbeck et al., 2009). It should be noted, however, that both studies, the

\section{REFERENCES}

Bardy, G. H., Lee, K. L., Mark, D. B., Poole, J. E., Packer, D. L., Boineau, R., Domanski, M., Troutman, C., Anderson, J., Johnson, G., McNulty, S. E., Clapp-Channing, N., Davidson-Ray, L. D., Fraulo, E. S., Fishbein, D. P., Luceri, R. M., and Ip, J. H. (2005). Amiodarone or an implantable cardioverterdefibrillator for congestive heart failure. N. Engl. J. Med. 352, 225-237.

Barthel, P., Bauer, A., Muller, A., Junk, N., Huster, K. M., Ulm, K., Malik, M.,

DINAMIT ( $n=674)$ and the IRIS study $(n=898)$, used entry criteria that selected only a very small proportion of post-infarction patients at very high risk. For instance, the IRIS study selected 898 patients out of 62,944 patients (1.4\%). Conclusions drawn from these two studies should therefore not be generalized and extrapolated to other risk stratification strategies.

\section{CONCLUSION}

Heart rate turbulence is easily applicable from routine 24-h Holter recordings. In all post-infarction studies HRT was a strong and independent predictor of adverse events which included death of any cause, cardiac death, and sudden death. In all post-infarction studies, predictive value of HRT was independent from other risk factors tested. For purpose of identifying high risk individuals who might benefit from prophylactic ICD implantation, HRT should be combined with other independent predictors. Potential candidates include impaired LVEF, abnormal deceleration capacity, and/or T-wave alternans. The combination of abnormal HRT and abnormal deceleration capacity termed "SAF" has been tested in the ISAR-RISK study and provides strong prognostic value also in post-infarction patients with preserved LV-function. However, only future interventional trials can finally answer the question whether high risk patients identified by abnormal HRT benefit from prophylactic therapy.

A., and Schmidt, G. (2003). Risk stratification after acute myocardial infarction by heart rate turbulence. Circulation 108, 1221-1226.

Bauer, A., Barthel, P., Schneider, R., Ulm, K., Muller, A., Joeinig, A., Stich, R., Kiviniemi, A., Hnatkova, K., Huikuri, H., Schomig, A., Malik, M., and Schmidt, G. (2009a). Improved stratification of autonomic regulation for risk prediction in postinfarction patients with preserved left ventricular function (ISAR-Risk). Eur. Heart J. 30, 576-583.

Bauer, A., Mehilli, J., Barthel, P., Muller, A., Kastrati, A., Ulm, K., Schomig, A., Malik, M., and Schmidt, G. (2009b). Impact of myocardial salvage assessed by (99m)Tc-sestamibi scintigraphy on cardiac autonomic function in patients undergoing mechanical reperfusion therapy for acute myocardial infarction. JACC Cardiovasc. Imaging 2, 449-457. 
Bauer, A., Barthel, P., Muller, A., Ulm, K., Huikuri, H., Malik, M., and Schmidt, G. (2009c). Risk prediction by heart rate turbulence and deceleration capacity in postinfarction patients with preserved left ventricular function retrospective analysis of 4 independent trials. J. Electrocardiol. 42, 597-601.

Bauer, A., Guzik, P., Barthel, P., Schneider, R., Ulm, K., Watanabe, M. A., and Schmidt, G. (2005). Reduced prognostic power of ventricular late potentials in post-infarction patients of the reperfusion era. Eur. Heart J. 26, 755-761.

Bauer, A., Kantelhardt, J. W., Barthel, P., Schneider, R., Makikallio, T., Ulm, K., Hnatkova, K., Schomig, A., Huikuri, H., Bunde, A., Malik, M., and Schmidt, G. (2006a). Deceleration capacity of heart rate as a predictor of mortality after myocardial infarction: cohort study. Lancet 367, 1674-1681.

Bauer, A., Watanabe, M., Barthel, P., Schneider, R., Ulm, K., and Schmidt, G. (2006b). QRS duration and late mortality in unselected postinfarction patients of the revascularisation era. Eur. Heart J. 27, 427-433.

Bauer, A., Kantelhardt, J. W., Bunde, A., Malik, M., Schneider, R., and Schmidt, G. (2006c). Phase-rectified signal averaging detects quasiperiodicities in non-stationary data. Physica A 364, 423-434.

Bauer, A., Malik, M., Schmidt, G., Barthel, P., Bonnemeier, H., Cygankiewicz, I., Guzik, P., Lombardi, F., Muller, A., Oto, A., Schneider, R., Watanabe, M., Wichterle, D., and Zareba, W. (2008). Heart rate turbulence: standards of measurement, physiological interpretation, and clinical use: International Society for Holter and Noninvasive Electrophysiology Consensus. J. Am. Coll. Cardiol. 52, 1353-1365.

Bauer, A., and Schmidt, G. (2007). Last piece of the heart rate turbulence puzzle? Heart Rhythm 4, 290-291.

Berkowitsch, A., Zareba, W., Neumann, T., Erdogan, A., Nitt, S. M., Moss, A. J., and Pitschner, H. F. (2004). Risk stratification using heart rate turbulence and ventricular arrhythmia in MADIT II: usefulness and limitations of a 10-minute Holter recording. Ann. Noninvasive Electrocardiol. 9, 270-279.

Bristow, M. R., Saxon, L. A., Boehmer, J., Krueger, S., Kass, D. A., De Marco, T., Carson, P., DiCarlo, L., DeMets, D., White, B. G., DeVries, D. W., and Feldman, A. M. (2004). Cardiacresynchronization therapy with or without an implantable defibrillator in advanced chronic heart failure. $N$. Engl. J. Med. 350, 2140-2150.

Buxton, A. E. (2003). Risk stratification for sudden death: do we need anything more than ejection fraction? Card. Electrophysiol. Rev. 7, 434-437.

Buxton, A. E., Lee, K. L., Fisher, J. D., Josephson, M. E., Prystowsky, E. N., and Hafley, G. (1999). A randomized study of the prevention of sudden death in patients with coronary artery disease. Multicenter Unsustained Tachycardia Trial Investigators. N. Engl. J. Med. 341, 1882-1890.

Camm, J., Klein, H., and Nisam, S. (2007). The cost of implantable defibrillators: perceptions and reality. Eur. Heart J. 28, 392-397.

Crenshaw, B. S., Ward, S. R., Granger, C. B., Stebbins, A. L., Topol, E. J., and Califf, R. M. (1997). Atrial fibrillation in the setting of acute myocardial infarction: the GUSTO-I experience. Global utilization of streptokinase and TPA for occluded coronary arteries. J. Am. Coll. Cardiol. 30, 406-413.

Exner, D. V., Kavanagh, K. M., Slawnych, M. P., Mitchell, L. B., Ramadan, D., Aggarwal, S. G., Noullett, C., Van Schaik, A., Mitchell, R. T., Shibata, M. A., Gulamhussein, S., McMeekin, J., Tymchak, W., Schnell, G., Gillis, A. M., Sheldon, R. S., Fick, G. H., and Duff, H. J. (2007). Noninvasive risk assessment early after a myocardial infarction the REFINE study. J. Am. Coll. Cardiol. 50, 2275-2284.

Ghuran, A., Reid, F., La Rovere, M. T., Schmidt, G., Bigger, J. T. Jr., Camm, A. J., Schwartz, P. J., and Malik, M. (2002). Heart rate turbulencebased predictors of fatal and nonfatal cardiac arrest (the autonomic tone and reflexes after myocardial infarction substudy). Am. J. Cardiol. 89, 184-190.

Gregoratos, G., Abrams, J., Epstein, A., Freedman, R., Hayes, D., Hlatky, M., Kerber, R., Naccarelli, G., Schoenfeld, M., Silka, M., and Winters, S. (2002). ACC/AHA/NASPE 2002 guideline update for implantation of cardiac pacemakers and antiarrhythmia devices - summary article: a report of the American College of Cardiology/American Heart Association Task Force on Practice Guidelines (ACC/AHA/NASPE Committee to update the 1998 pacemaker guidelines). J. Am. Coll. Cardiol. 40, 1531-1540.
Hallstrom, A. P., Stein, P. K., Schneider, R., Hodges, M., Schmidt, G. and Ulm, K. (2005). Characteristics of heart beat intervals and prediction of death. Int. J. Cardiol. 100, 37-45.

Hohnloser, S. H., Kuck, K. H., Dorian, P., Roberts, R. S., Hampton, J. R., Hatala, R., Fain, E., Gent, M., and Connolly, S. J. (2004). Prophylactic use of an implantable cardioverterdefibrillator after acute myocardial infarction. N. Engl. J. Med. 351, 2481-2488.

Huikuri, H. V., Castellanos, A., and Myerburg, R. J. (2001). Sudden death due to cardiac arrhythmias. $N$. Engl. J. Med. 345, 1473-1482.

Huikuri, H. V., Exner, D. V., Kavanagh, K. M., Aggarwal, S. G., Mitchell, L. B., Messier, M. D., Becker, D., Sheldon, R. S., and Bloch Thomsen, P. E. (2010). Attenuated recovery of heart rate turbulence early after myocardial infarction identifies patients at high risk for fatal or near-fatal arrhythmic events. Heart Rhythm 7, 229-235.

Huikuri, H. V., Raatikainen, M. J., Moerch-Joergensen, R., Hartikainen, J., Virtanen, V., Boland, J., Anttonen, O., Hoest, N., Boersma, L. V., Platou, E. S., Messier, M. D., and Bloch-Thomsen, P. E. (2009). Prediction of fatal or near-fatal cardiac arrhythmia events in patients with depressed left ventricular function after an acute myocardial infarction. Eur. Heart J. 30, 689-698.

Huikuri, H. V., Tapanainen, J. M., Lindgren, K., Raatikainen, P., Makikallio, T. H., Juhani Airaksinen, K. E., and Myerburg, R. J. (2003). Prediction of sudden cardiac death after myocardial infarction in the betablocking era. J. Am. Coll. Cardiol. 42, 652-658.

La Rovere, M. T., Bigger, J. T. Jr., Marcus, F. I., Mortara, A., and Schwartz, P. J. (1998). Baroreflex sensitivity and heart-rate variability in prediction of total cardiac mortality after myocardial infarction. ATRAMI (autonomic tone and reflexes after myocardial infarction) investigators. Lancet 351, 478-484.

Makikallio, T. H., Barthel, P., Schneider, R., Bauer, A., Tapanainen, J. M., Tulppo, M. P., Schmidt, G. and Huikuri, H. V. (2005). Prediction of sudden cardiac death after acute myocardial infarction: role of Holter monitoring in the modern treatment era. Eur. Heart J. 26 , 762-769.

Moss, A. J., Hall, W. J., Cannom, D. S., Daubert, J. P., Higgins, S. L., Klein, H., Levine, J. H., Saksena, S., Waldo,
A. L., Wilber, D., Brown, M. W. and Heo, M. (1996). Improved survival with an implanted defibrillator in patients with coronary disease at high risk for ventricular arrhythmia. Multicenter Automatic Defibrillator Implantation Trial Investigators. N. Engl. J. Med. 335, 1933-1940.

Moss, A. J., Zareba, W., Hall, W. J., Klein, H., Wilber, D. J., Cannom, D. S., Daubert, J. P., Higgins, S. L., Brown, M. W., and Andrews, M. L. (2002). Prophylactic implantation of a defibrillator in patients with myocardial infarction and reduced ejection fraction. N. Engl. J. Med. 346, 877-883.

Myerburg, R. J., Interian, A. Jr., Mitrani, R. M., Kessler, K. M., and Castellanos, A. (1997). Frequency of sudden cardiac death and profiles of risk. Am. J. Cardiol. 80, 10F-19F.

Myerburg, R. J., Mitrani, R., Interian, A. Jr., and Castellanos, A. (1998). Interpretation of outcomes of antiarrhythmic clinical trials: design features and population impact. Circulation 97, 1514-1521.

Schmidt, G., Malik, M., Barthel, P., Schneider, R., Ulm, K., Rolnitzky, L., Camm, A. J., Bigger, J. T. Jr., and Schomig, A. (1999). Heart-rate turbulence after ventricular premature beats as a predictor of mortality after acute myocardial infarction. Lancet 353, 1390-1396.

Segerson, N. M., Wasmund, S. L., Abedin, M., Pai, R. K., Daccarett, M., Akoum, N., Wall, T. S., Klein, R. C., Freedman, R. A., and Hamdan, M. H. (2007). Heart rate turbulence parameters correlate with post-premature ventricular contraction changes in muscle sympathetic activity. Heart Rhythm 4, 284-289.

Stein, P. K., Sanghavi, D., Sotoodehnia, N., Siscovick, D. S., and Gottdiener, J. (2010). Association of Holter-based measures including Twave alternans with risk of sudden cardiac death in the communitydwelling elderly: the Cardiovascular Health Study. J. Electrocardiol. 43, 251-259.

Steinbeck, G., Andresen, D., Seidl, K., Brachmann, J., Hoffmann, E., Wojciechowski, D., Kornacewicz-Jach Z., Sredniawa, B., Lupkovics, G., Hofgartner, F., Lubinski, A., Rosenqvist, M., Habets, A., Wegscheider, K., and Senges, J. (2009). Defibrillator implantation early after myocardial infarction. N. Engl. J. Med. 361, 1427-1436.

Wichterle, D., Melenovsky, V., Simek, J., Malik, J., and Malik, M. (2006). 
Hemodynamics and autonomic control of heart rate turbulence. J. Cardiovasc. Electrophysiol. 17, 286-291.

Zipes, D. P., Camm, A. J., Borggrefe, M., Buxton, A. E., Chaitman, B., Fromer, M., Gregoratos, G., Klein, G., Moss, A. J., Myerburg, R. J., Priori, S. G., Quinones, M. A., Roden, D. M., Silka, M. J., Tracy, C., Smith, S. C. Jr., Jacobs, A. K., Adams, C. D., Antman, E. M., Anderson, J. L., Hunt, S. A., Halperin, J. L., Nishimura, R., Ornato, J. P., Page, R. L., Riegel, B., Blanc, J. J., Budaj, A., Dean, V., Deckers, J. W., Despres, C., Dickstein, K.,
Lekakis, J., McGregor, K., Metra, M., Morais, J., Osterspey, A., Tamargo, J. L., and Zamorano, J. L. (2006). ACC/AHA/ESC 2006 guidelines for management of patients with ventricular arrhythmias and the prevention of sudden cardiac death: a report of the American College of Cardiology/American Heart Association Task Force and the European Society of Cardiology Committee for Practice Guidelines (writing committee to develop guidelines for management of patients with ventricular arrhythmias and the prevention of sudden cardiac death): developed in collaboration with the European Heart Rhythm Association and the Heart Rhythm Society. Circulation 114, e385-e484.

Conflict of Interest Statement: The authors declare that the research was conducted in the absence of any commercial or financial relationships that could be construed as a potential conflict of interest.

Received: 02 September 2011; paper pending published: 06 October 2011; accepted: 24 November 2011; published online: 12 December 2011.
Citation: Zuern CS, Barthel $P$ and Bauer A (2011) Heart rate turbulence as risk-predictor after myocardial infarction. Front. Physio. 2:99. doi: 10.3389/fphys.2011.00099

This article was submitted to Frontiers in Clinical and Translational Physiology, a specialty of Frontiers in Physiology. Copyright (c) 2011 Zuern, Barthel and Bauer. This is an open-access article distributed under the terms of the Creative Commons Attribution Non Commercial License, which permits non-commercial use, distribution, and reproduction in other forums, provided the original authors and source are credited. 